\title{
The effect of vacancy-induced magnetism on electronic transport in armchair carbon nanotubes
}

\author{
R. Farghadan ${ }^{1}$ and A. Saffarzadeh ${ }^{2,3, *}$ \\ ${ }^{I}$ Department of Physics, Tarbiat Modares University, Tehran, Iran \\ ${ }^{2}$ Department of Physics, Payame Noor University, Nejatollahi Street, 159995-7613 Tehran, Iran \\ ${ }^{3}$ Computational Physical Sciences Laboratory, Department of Nano-Science, \\ Institute for Research in Fundamental Sciences (IPM), P.O. Box 19395-5531, Tehran, Iran
}

(Dated: October 4, 2018)

\begin{abstract}
The influence of local magnetic moment formation around three kinds of vacancies on the electron conduction through metallic single-wall carbon nanotubes is studied by use of the Landauer formalism within the coherent regime. The method is based on the single-band tight-binding Hamiltonian, a surface Green's function calculation, and the mean-field Hubbard model. The numerical results show that the electronic transport is spin-polarized due to the localized magnetic moments and it is strongly dependent on the geometry of the vacancies. For all kinds of vacancies, by including the effects of local magnetic moments, the electron scattering increases with respect to the nonmagnetic vacancies case and hence, the current-voltage characteristic of the system changes. In addition, a high value for the electron-spin polarization can be obtained by applying a suitable gate voltage.

PACS numbers: 72.10.-d, 72.10.Fk
\end{abstract}

\section{INTRODUCTION}

The electrical transport properties of single-wall carbon nanotubes (CNTs) and other carbon-based materials have attracted much attention due to their unusual properties and great potential for technological applications [1-4]. Among these features, the ballistic electron conduction and the long range spin coherent transport for perfect and defective singlewall CNTs have been investigated theoretically and experimentally [5-7]. These important properties of spin polarized electrons in single-wall CNTs has motivated their use in the emerging field of spin electronics [8] which aims to effectively control and manipulate the spin degrees of freedom in the electronic devices [9].

In order to achieve spintronic devices based on single-wall CNTs, it is important to understand the magnetic effect of vacancies and impurities on the electron conduction [8, 10, 11]. because, the electronic properties of carbon-based materials strongly depend on their topological structure [12]. Therefore the electronic structure of the CNTs can differ due to the topological defects or the addition of different compounds. On the other hand, in the transport processes, the ballistic conductance depends on the number of conducting channels at the Fermi energy [13]. Consequently, the appearance of the vacancy defects in a structure can change the electronic and transport properties of the system [5]. Also, the conductance of an imperfect system is lowered due to the reflection of electron waves from the defects [14]. In addition, the localized states near the vacancy are magnetic and change the net magnetic moments in the carbon-based nanostructures. The honeycomb lattice of graphene sheet is formed by two sublattices $A$ and $B$ (bipartite lattice). For a bipartite lattice with different numbers $N_{A}$ and $N_{B}$ of sites and the Hubbard repulsive parameter, the total spin, $S$, of the ground state of the system, which is mainly localized near the vacancy, is $2 S=N_{A}-N_{B}$ [15]. This important feature can block and change the spin transport especially near the Fermi energy.

In this regard, the electronic and magnetic properties of vacancies in single-wall CNTs [16] and graphene nanoribbons [17] have been investigated. In addition, the electronspin polarization has been observed in the CNTs when doped with magnetic adatoms or molecules [18]. Moreover, the localized states of the impurity can change the spin-polarized conduction in the presence of a gate voltage or applied bias [8, 19]. Recently, the effect of vacancy on the conductance of single-wall CNTs [20, 21] and the spin-dependent transport properties in ferromagnetically contacted single-wall CNTs have been investigated [19, 22, 23], but the magnetic behavior of vacancy defect as regards the transmission of single-wall CNTs has not been considered.

The purpose of this work is to study the effect of magnetic vacancies on the spin-polarized transport through armchair CNT junctions and manipulate this polarization by means of gate voltages. We simulate ideal vacancies which are made by removing carbon atoms from lattice sites without including the deformation of the tube wall around the vacancies. We consider three typical vacancy types. For the first type, a single carbon site (A or B) is removed. For the second one, two same sites (two A or B sites), and in the last type, four A sites in one carbon ring are removed, as shown in Figs. 1(a) and 1(b), respectively.

Using the single-band tight-binding approximation and the mean-field Hubbard model [24], the electronic structure and the localized magnetic moments around the above-mentioned vacancies are calculated. Also, using the non-equilibrium Green's function technique and the Landauer-Büttiker theory [25], the spin-polarized transport in defective single-wall CNTs is investigated. 
(a)

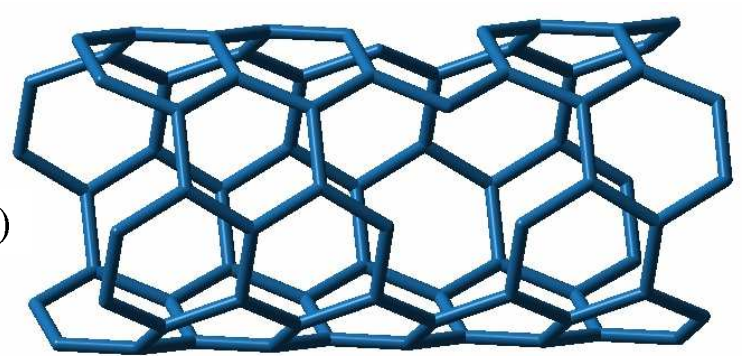

(b)

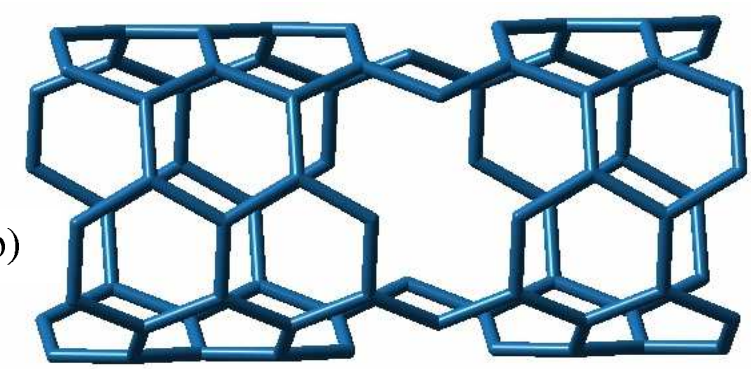

FIG. 1: Five unit cells of a $(4,4)$ single-wall CNT (consist of 80 atoms) as the channel, in the presence of (a) two and (b) four vacancies on a ring of carbon atoms.

\section{MODEL AND FORMALISM}

We consider a system that consists of a defective CNT with finite length, sandwiched between two perfect semi-infinite CNTs as electrodes. All CNTs have same structure, $(n, n)$. To calculate the electron transmission properties of this defective CNT which acts as a channel, the electronic structure of this system should be resolved in detail. Hence, we decompose the total Hamiltonian of the system as [26]

$$
\hat{H}=\hat{H}_{L}+\hat{H}_{C}+\hat{H}_{R}+\hat{H}_{T},
$$

where $\hat{H}_{L}$ and $\hat{H}_{R}$ are the Hamiltonians of the left $(L)$ and right $(R)$ electrodes, $\hat{H}_{C}$ describes the channel Hamiltonian and contains the magnetic properties of the vacancies, and $\hat{H}_{T}$ is the coupling between the electrodes and the central part (defective CNT). The total Hamiltonian is described within the single-band tight-binding approximation. Therefore, the Hamiltonian of the electrodes can be written as [27]

$$
\hat{H}_{\alpha}=\sum_{<i_{\alpha}, j_{\alpha}>, \sigma}\left(\epsilon_{\alpha} \delta_{i_{\alpha}, j_{\alpha}}-t_{i_{\alpha}, j_{\alpha}}\right) \hat{c}_{i_{\alpha}, \sigma}^{\dagger} \hat{c}_{j_{\alpha}, \sigma}
$$

where $\hat{c}_{i_{\alpha}, \sigma}^{\dagger}\left(\hat{c}_{i_{\alpha}, \sigma}\right)$ creates (destroys) an electron at site $i$ in electrode $\alpha(=R, L)$ and the hopping elements $t_{i_{\alpha}, j_{\alpha}}$ are equal to $t$ for nearest neighbors and zero otherwise. Here, $\epsilon_{\alpha}$ is the on-site energy of the electrodes and will be set to zero. The coupling Hamiltonian is described as

$$
\hat{H}_{T}=-\sum_{\alpha=\{L, R\}} \sum_{i_{\alpha}, j_{C}, \sigma} t_{i_{\alpha}, j_{C}}\left(\hat{c}_{i_{\alpha}, \sigma}^{\dagger} \hat{d}_{j_{C}, \sigma}+\text { H.c. }\right)
$$

The parameters $t_{i_{\alpha}, j_{C}}$ for hopping between the electrodes and the channel are taken to be $t$. In order to obtain the magnetic moment of each atom around a vacancy, we use the Hubbard model within the unrestricted Hartree-Fock approximation [24]. Accordingly, the Hamiltonian of the central part within the mean-field approximation of the Hubbard model can be written as [24, 28]

$$
\begin{array}{r}
\hat{H}_{C}=\sum_{i \sigma} \epsilon_{i} \hat{d}_{i \sigma}^{\dagger} \hat{d}_{i \sigma}-t \sum_{i, j, \sigma} \hat{d}_{i \sigma}^{\dagger} \hat{d}_{j \sigma}+U \sum_{i}\left\{\left\langle\hat{n}_{i \uparrow}\right\rangle \hat{n}_{i \downarrow}\right. \\
\left.+\left\langle\hat{n}_{i \downarrow}\right\rangle \hat{n}_{i \uparrow}-\left\langle\hat{n}_{i \uparrow}\right\rangle\left\langle\hat{n}_{i \downarrow}\right\rangle\right\},
\end{array}
$$

where $\epsilon_{i}$ is the on-site energy and will be set to the gate potential in the channel region, the second term corresponds to the single $\pi$-orbital tight-binding Hamiltonian, while the third term accounts for the on-site Coulomb interaction $U$. In this expression, $\hat{d}_{i \sigma}^{\dagger}\left(\hat{d}_{i \sigma}\right)$ creates (annihilates) an electron, and $\left\langle\hat{n}_{i \sigma}\right\rangle$ is the expectation value of the number operator for an electron with spin $\sigma$ at the $i$ th site.

The Green's function of the system can be written as

$$
\hat{G}_{C}(\varepsilon)=\left[\varepsilon \hat{I}-\hat{H}_{C}-\hat{\Sigma}_{L}(\varepsilon)-\hat{\Sigma}_{R}(\varepsilon)\right]^{-1},
$$

where $\hat{\Sigma}_{\alpha}(\varepsilon)=\hat{H}_{C \alpha} \hat{g}_{\alpha}(\varepsilon) \hat{H}_{C \alpha}^{\dagger}$ is the self-energy matrix which contains the influence of the electronic structure of the semi-infinite electrodes through the lead's surface Green's function $g_{\alpha}[29,30]$. Correspondingly, $\hat{H}_{C \alpha}$ defines the matrix of coupling between the surface atomic orbitals of the lead $\alpha$ and the channel. We should note that, $\hat{G}_{C}$ is a spindependent matrix of size $2 N$, with $N$ being the number of atoms of the central region. Therefore, due to our mean-field decoupling scheme, we can decouple the electronic transport into spin-up and spin-down currents.

Using the iterative method for the calculation of the transfer matrices based on the principal layers concept, the surface Green's function of the left and right leads can be obtained as [29]:

$$
\begin{gathered}
\hat{g}_{L}(\varepsilon)=\left[\varepsilon \hat{I}-\hat{H}_{00}^{L}-\hat{H}_{01}^{L} \hat{\bar{T}}_{L}\right], \\
\hat{g}_{R}(\varepsilon)=\left[\varepsilon \hat{I}-\hat{H}_{00}^{R}-\hat{H}_{01}^{R} \hat{T}_{R}\right],
\end{gathered}
$$

where $\hat{H}_{00}^{\alpha}$ and $\hat{H}_{01}^{\alpha}$ are matrices and correspond to an isolated principal layer and the interaction between two nearest principle layers in lead $\alpha$, respectively. Also, $\hat{\bar{T}}_{L}$ and $\hat{T}_{R}$ are the transfer matrices and for the details of the calculations the reader can refer to Ref [29]. From the expression of the nonequilibrium Green's function, the spin-dependent local density of states (LDOS) and the expectation value for the number operator of electrons in the channel are given by

$$
D_{i \sigma}(\varepsilon)=-\frac{1}{\pi} \operatorname{Im}\left(\left\langle i \sigma\left|\hat{G}_{C}(\varepsilon)\right| i \sigma\right\rangle\right),
$$

$$
\left\langle\hat{n}_{i \sigma}\right\rangle=\int_{-\infty}^{E_{F}} D_{i \sigma}(\varepsilon) d \varepsilon
$$


Accordingly, the magnetic moment, $m_{i}$, at site $i$ of the channel can be calculated using

$$
m_{i}=\mu_{B}\left[\left\langle\hat{n}_{i \uparrow}\right\rangle-\left\langle\hat{n}_{i \downarrow}\right\rangle\right] .
$$

In this study, we solve the mean field Hamiltonian selfconsistently by an iterative method [24]. In the first step, we start from an anti-ferromagnetic configuration as an initial condition and establish the Hamiltonian for the Hubbard model [Eq. [4]]. In the second step, the effect of electrodes on the channel is added via $\hat{\Sigma}_{L}$ and $\hat{\Sigma}_{R}$, and then the Green's function of the channel, $\hat{G}_{C}$, in the presence of the electrodes is calculated. In the third step, the expectation values of the number operators $\left\langle\hat{n}_{i \sigma}\right\rangle$ at each site and for both spin alignments are calculated by using the Green's function. Finally the new expectation values of the number operators are replaced in Eq. (4), and this process is repeated until the difference between two successive iterations becomes less than $10^{-4}$.

The total Hamiltonian does not contain inelastic scattering, because there is no spin-flip or the other sources of scattering in the system. In other words, the transmission probabilities of majority and minority subbands are independent and the electronic transport can be decoupled into two spin currents: one for spin-up and the other for spin-down. Therefore, the spindependent currents for a constant bias voltage are calculated by using the Landauer-Büttiker formalism [10, 26]:

$$
I_{\sigma}\left(V_{a}\right)=\frac{e}{h} \int_{-\infty}^{\infty} T_{\sigma}(\varepsilon)\left[f\left(\varepsilon-\mu_{L}\right)-f\left(\varepsilon-\mu_{R}\right)\right] d \varepsilon,
$$

where $f$ is the Fermi-Dirac distribution function, $\mu_{L, R}=$ $E_{F} \pm \frac{1}{2} e V_{a}$ are the chemical potentials of the electrodes and $T_{\sigma}(\varepsilon)=\operatorname{Tr}\left[\hat{\Gamma}_{L} \hat{G}_{C, \sigma} \hat{\Gamma}_{R} \hat{G}_{C, \sigma}^{\dagger}\right]$ is the energy- and spindependent transmission function. Using $\hat{\Sigma}_{\alpha}$, the coupling matrices $\hat{\Gamma}_{\alpha}$ can be expressed as $\hat{\Gamma}_{\alpha}=-2 \operatorname{Im}\left[\hat{\Sigma}_{\alpha}(\varepsilon)\right]$.

\section{RESULTS AND DISCUSSION}

We study the LDOS, the transmission and the currentvoltage characteristic of the single-wall CNTs in the presence of vacancies when the CNT in all regions is of $(4,4)$ type. We expect the magnetic behavior of the vacancy to affect the electron conduction through the structure, since different scatterings occur for the electronic waves with different spin densities. Therefore, the transmission for majority electrons can be different from that of the minority electrons at certain energies.

In the cases of the first, second and third vacancy types, the total magnetic moments for the finite CNT (channel) as shown in Fig. 1, are 1,2 and $4 \mu_{B}$ respectively, which are in good agreement with the Lieb's theorem [15]. However, when the two semi-infinite CNTs (electrodes) are attached to the channel, their electronic properties are changed. This causes appreciable variation in the value of magnetic moment of each atomic site near the vacancies and affects the spin-dependent

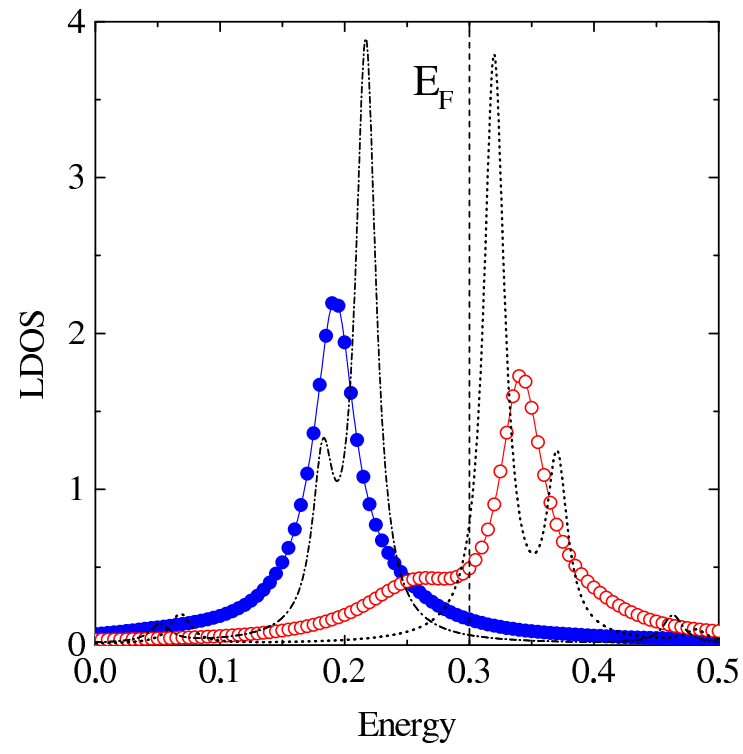

FIG. 2: LDOS per site around two vacancies for the CNT/defectiveCNT/CNT junction with $U=1.06 t, V_{G}=0.0 t$, and $V_{a}=0.0 t$. Full (open) circles are for majority (minority) spin electron. The dotted (dashed-dotted) line is for majority (minority) spin in the absence of CNT leads ( and the dashed line indicates the Fermi energy). Note that, the majority and minority electrons are determined by Eq. (7).

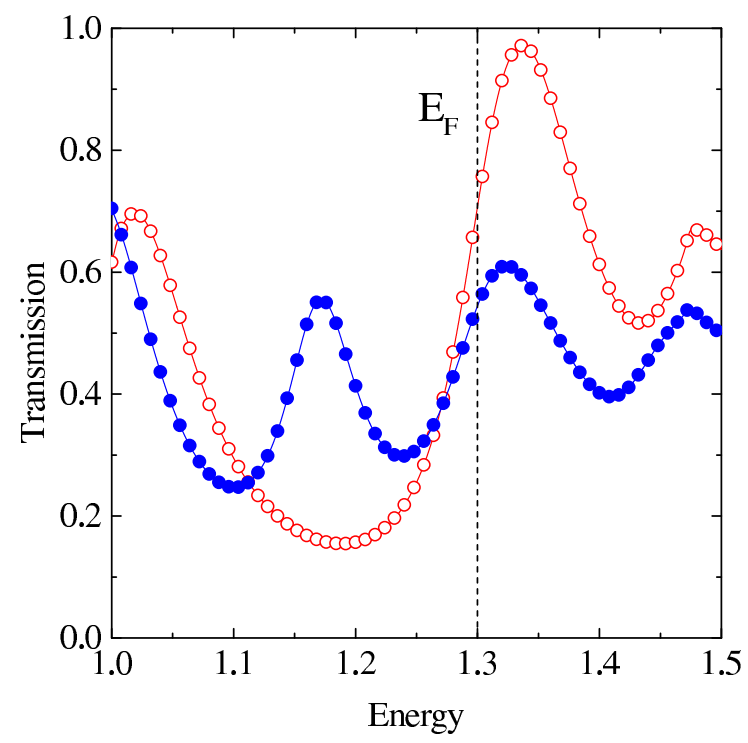

FIG. 3: Transmission coefficients as a function of energy in the presence of two vacancies (second type) with $V_{G}=1.0 t$ and $V_{a}=0.0 t$. Full (open) circles are for majority (minority) spin electrons.

transport through the channel. When the atoms from the same sublattice are removed, the final spin configuration, after selfconsistent calculations for the atomic sites near the vacancies, is ferromagnetic. These magnetic moments only obtain for the atomic sites near the vacancies and for the other sites approximately vanish. Our numerical results showed that in the presence of a single vacancy, the magnetic moment vanishes approximately and an unpolarized ground state is obtained for 
the value of $U=1.06 t$, due to the effect of semi-infinite electrodes on the channel [31].

In order to clarify this effect, we have plotted in Fig. 2 the LDOS in the presence of two vacancies. In a defective $\mathrm{CNT}$, the electronic states on the carbon atoms near the vacancy strongly depend on the presence or absence of CNT electrodes. In a finite-length CNT, the electronic states on carbon atoms are almost localized due to the absence of periodicity along the axis of CNT. Therefore, sharp features are observed in the spin-dependent LDOS for the states around the Fermi energy. When the electrodes are attached to the defective CNT, the localization of electronic states is suppressed and the sharp peaks are broadened for both majority and minority electrons. Accordingly, the difference between the two densities of states and hence the spin polarizations are reduced. Note that in both cases the condition $\left\langle\hat{n}_{i \uparrow}\right\rangle+\left\langle\hat{n}_{i \downarrow}\right\rangle=1$ at each site is satisfied.

It should be pointed out that the Hubbard repulsive parameter shifts the Fermi energy from zero to $E_{F}=0.3 t$ as shown with a vertical line in Fig. 2. In fact, when the two vacancies in the channel are included in the calculations, we obtain $S=0.55$ for the total spin value in the presence of CNT electrodes and the maximum value of the magnetic moment reaches $0.16 \mu_{B}$. This magnetic moment can affect the spin transport in the channel.

To see the effects of vacancy-induced magnetism on the electron conduction, we have shown in Fig. 3 the transmission coefficients as a function of energy for majority and minority electrons at $V_{G}=1.0 t$. The transmission spectrum of two spin subbands is non-degenerate for all electronic states within the energy window and there is a significant difference between the two spin subbands. Since in the process of electronic transport, the electrons with Fermi energy carry most of the current, we have shown the transmission coefficients around the Fermi level. It is clear that the Fermi energy shifts from $0.3 t$ to $1.3 t$ due to the gate voltage.

We note that a gate voltage shifts the electronic states and hence, the transmission for two spin subbands in the central region can vary significantly. Therefore, a gate potential is able to change the spin transport through such a defective CNT. In order to see the effect of the gate voltage on the spin transport, we have calculated the degree of spin polarization $(P)$ for electrons traversing the channel which can be defined as $P(E)=\frac{T_{\uparrow}(E)-T_{\downarrow}(E)}{T_{\uparrow}(E)+T_{\downarrow}(E)}$. Fig. 4 shows this physical quantity in terms of the gate voltage in the case of two vacancies. It is clear that the spin polarization can reach values as high as $65 \%$. Also, we found that the change in the position of the two vacancies with respect to each other causes appreciable variations in the value of the localized magnetic moment and hence, in the degree of spin-polarization. Generally, on increasing the distance between two vacancies the total value of the channel magnetic moment decreases.

Fig. 5. shows the transmission coefficients as a function of energy for majority and minority electrons in the case of four vacancies (third type). Only the energy window around

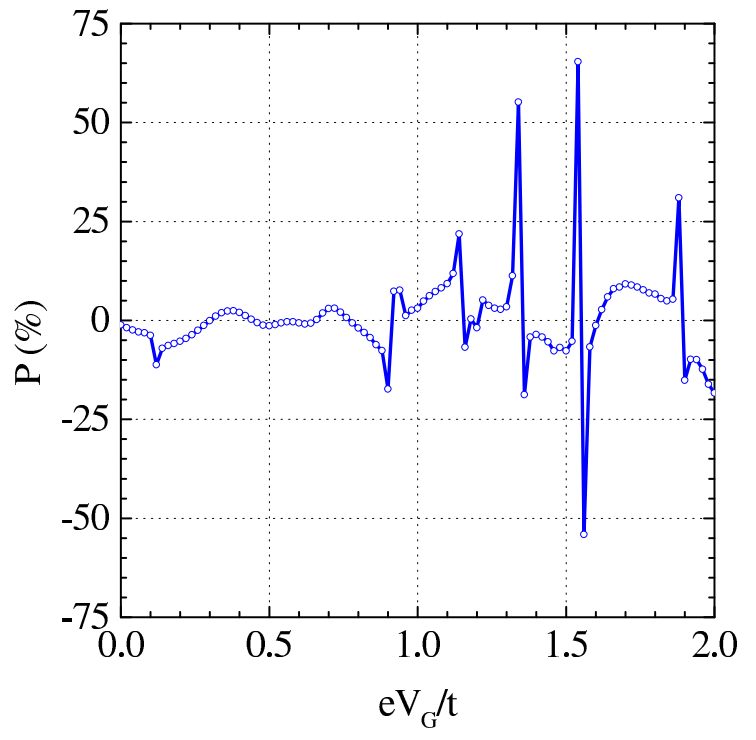

FIG. 4: Degree of spin polarization as a function of gate voltage for vacancies of the second type at the Fermi energy and $V_{a}=0.0 t$.

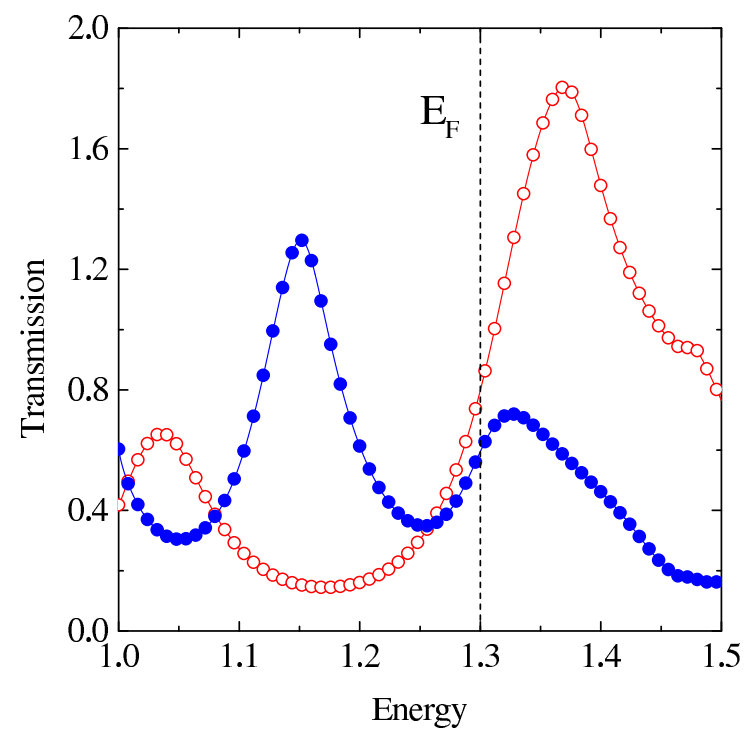

FIG. 5: Transmission coefficients as a function of energy in the presence of four vacancies (third type), for $V_{G}=1.0 t$ and $V_{a}=0.0 t$. Full (open) circles are for majority (minority) spin electrons.

the Fermi level has been shown $\left(E_{F}=1.3 t\right)$. As we know, in the present structure where the electrodes are made of CNTs, there are more paths for the electrons to pass from the electrodes to the defective CNT. Therefore, in some energy ranges of this figure, the spin-dependent transmission coefficients can be larger than unity. The total value of the spin is 1.40 and the maximum value of the magnetic moment reaches $0.30 \mu_{B}$. Also the maximum value of the spin polarization at $V_{G}=1.0 t$, reaches $80 \%$ which indicates that the local magnetism induced by vacancies can be useful in spintronic devices. This spin polarization of the electrical conductivity can play an important role in the current-voltage characteristics in 
the channel.

For this reason and to further understand the effect of vacancy-induced magnetism, we have plotted in Fig. 6 the spin currents in terms of the applied voltage. Since the electron conduction depends on the electronic states lying between $\mu_{L}$ and $\mu_{R}$, on increasing the applied voltage, these states move inside the energy window and the electronic currents for both majority and minority spin electrons increase. From the current-voltage characteristic, we see that the applied voltage can change the difference between both spin-up and spin-down currents significantly and therefore, we should adjust the value of the gate and applied voltages to obtain maximum values for the degree of polarization of the spin currents.

In this study, we only focused on the A-site defects because according to Lieb's theorem, when the same sublattice atoms are removed the total magnetic moment increases and therefore the polarization in the spin currents becomes remarkable. Also, our numerical results showed that if we remove two different sites from one carbon ring, the local magnetic moments near the vacancies are nonzero, while the total magnetic moment is zero and hence, the transmission coefficients of two spin channels are degenerate and practically unpolarized. In other cases, i.e., when different numbers of A and B sites are removed, the total magnetic moment is small and a little spin polarization can be observed (not shown here).

\section{CONCLUSION}

In this work, the coherent spin-polarized transport through a CNT/defective-CNT/CNT junction was investigated on the basis of the non-equilibrium Green's function technique, and the mean-field Hubbard model. We have shown that there are major differences in the behavior of the electronic transport when different kinds of vacancies are considered. The numerical results indicate that, the point defects of which different types were considered-and hence with different magnetic responses-could effectively alter the electron conduction through the junction.

In the defective CNTs, the difference between the transmission coefficients of two spin subbands at some energy values is small. Consequently, in such a case, the degree of spin polarization can be manipulated by applying a gate voltage to the channel. In addition, our results suggest that by choosing the position of the vacancy in the channel properly to enhance the local magnetization, one can increase the values of the spinpolarized currents. These defects may be useful in the process of spin injection into semiconducting devices for spintronic applications.

Throughout the study, we have ignored the effect of spinflip scattering, especially near the vacancies. This factor may affect the spin-dependent transport, and hence, a further improved approach is needed to obtain more accurate results.

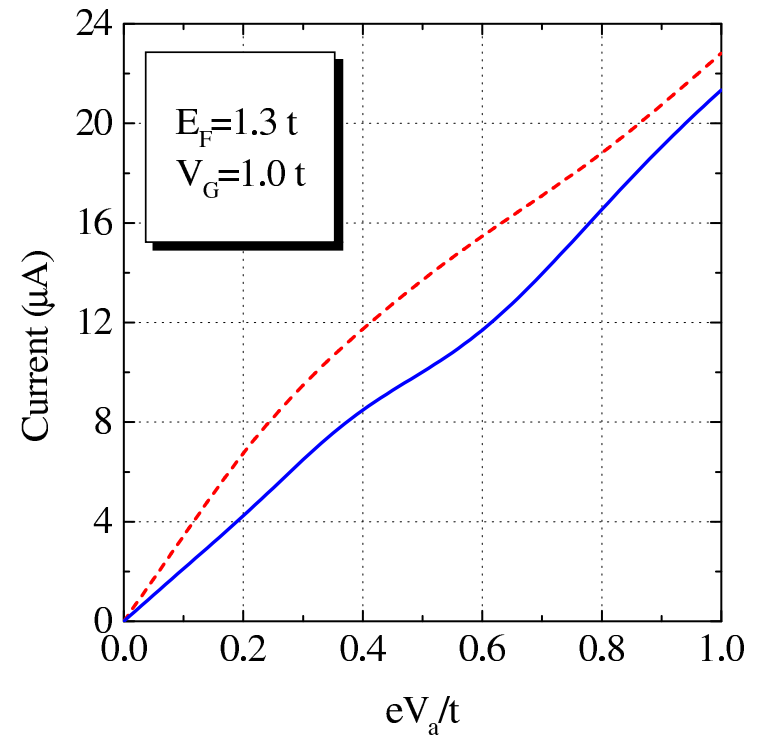

FIG. 6: Current-voltage characteristics in the presence of four vacancies. The solid (dashed) line is for majority (minority) spin electrons.

\section{Acknowledgement}

The authors would like to thank J. J. Palacios, K. Wakabayashi, M. A. H. Vozmediano, and M. B. Nardelli for helpful discussions.

* Electronic address: a-saffar@tpnu.ac.ir

[1] Nardelli M B 1999 Phys. Rev. B 607828

[2] Rochefort A, Avouris P, Lesage F and Salahub D R 1999 Phys. Rev. B 6013824

[3] Berger C et al. 2006 Science 3121191.

[4] Suarez V M G, Ferrer J and Lambert C J 2006 Phys. Rev. Lett. 96106804

[5] Ando T 2005 J. Phys. Soc. Jap. 74777

[6] Frank S, Poncharal P, Wang Z L and de Heer W A 1998 Science 2801744

[7] Tsukagoshi K, Alphenaar B W and Ago H 1999 Nature (London) 401572

[8] Son Y W, Cohen M L and Louie S G 2007 Nano Lett. 73518

[9] Zutic I, Fabian J and Sarma S D 2004 Rev. Mod. Phys. 76323

[10] Lehtinen P O, Foster A S, Ma Y, Krasheninnikov A V and Nieeminen R M 2004 Phys. Rev. Lett. 93187202

[11] Sancho M P L, Juan F D and Vozmediano M A H 2009 Phys. Rev. B 79075413

[12] Igami M, Nakanishi T and Ando T 1999 J. Phys. Soc. Jap 68 716

[13] Fisher D S and Lee P A 1981 Phys. Rev. B 236851

[14] Chico L, Benedict L X, Louie S G and Cohen M L 1996 Phys. Rev. B 542600

[15] Lieb E H 1989 Phys. Rev. Lett. 621201

[16] Orellana W and Fuentealba P 2006 Sur. Sci. 6004305

[17] Kumazaki H and Hirashima D S 2009 J. Phys. Soc. Jap 78 094701

[18] Yang C K, Zhao J and Lu J P 2004 Nano Lett. 4561 
[19] Fedorov G, Lassabne B, Sagnes M, Raquet B, Broto J M, Triozon F, Roche S and Flahaut E 2005 Phys. Rev. Lett. 94066801

[20] Choi H J and Ihm J 2000 Phys. Rev. Lett. 842917

[21] He Y, Zhang C, Cao C and Cheng H P 2007 Phys. Rev. B 75 235429.

[22] Kim J R, So H M and Kim J J 2002 Phys. Rev. B 66233401

[23] Jensen A, Hauptmann J R, Nygard J and Lindelof P E 2005 Phys. Rev. B 72035419

[24] Fujita M, Wakabayashi K and Nakada K 1996 J. Phys. Soc. Jap. 651920

[25] Datta S 2005 Quantum Transport: atom to transistor, (Cambridge University Press, Cambridge, England
[26] Saffarzadeh A 2008 J. Appl. Phys. 104123715

[27] Esfarjani K, Farajian A A, Ebrahimi F and Kawazoe K 2001 Eur. Phys. J. D 16353

[28] Rossier J F and Palacios J J 2007 Phys. Rev. Lett. 99177204

[29] López Sancho M P, López Sancho J M, and Rubio J 1984 J. Phys. F 141205

[30] Lee D H and Joannopoulos J D 1981 Phys. Rev. B 234988

[31] Rojas F M, Rossier J F, and Palacios J J 2009 Phys, Rev. Lett. 102136810 